\title{
Collaural Fistula: A Diagnostic Challenge
}

\author{
${ }^{1}$ Sachana Prasad, ${ }^{2}$ Amitabha Roychoudhury, ${ }^{3}$ Soumitra Ghosh
}

\section{ABSTRACT}

Aim: To emphasize on a comprehensive approach for correct diagnosis and treatment of first branchial cleft anomalies.

Background: First branchial cleft anomalies are congenital malformations accounting for less than $8 \%$ of all branchial cleft anomalies.

Case Description: We report a case of a 55-year-old female with a discharging collaural fistula that was misdiagnosed earlier and treated with repeated incision and drainage. Diagnosis was confirmed by history and CT fistulogram and the lesion was completely excised with no further recurrences.

Conclusion: Due to the varied presentations of the first branchial cleft defects, a compartmentalized approach by the clinician may lead to misdiagnosis and incorrect treatment.

Clinical Significance: The rare occurrence and diverse clinical presentations of these embryological defects have led to frequent delay in diagnosis as well as inadequate treatment. Correct diagnosis at primary presentation should be advocated for such rare entities.

Keywords: Branchial cleft anomalies, Collaural fistula, CT fistulogram

How to cite this article: Prasad S, Roychoudhury A, Ghosh S. Collaural Fistula: A Diagnostic Challenge. Int J Head Neck Surg 2017;8(4):153-156.

Source of support: Nil

Conflict of interest: None

\section{INTRODUCTION}

First branchial cleft anomalies are rare embryological defects accounting for less than $8 \%$ of all branchial anomalies. ${ }^{1,2}$ Diverse clinical manifestations of these anomalies in the form of cystic swelling, discharging sinuses and fistulas have been observed in the preauricular and cervical region above the level of hyoid bone.

${ }^{1}$ Post Graduate Trainee, ${ }^{2}$ Professor and Head, ${ }^{3}$ Associate Professor

${ }^{1-3}$ Department of Otorhinolaryngology, Vivekananda Institute of Medical Sciences, Kolkata, West Bengal, India

Corresponding Author: Sachana Prasad, Post Graduate Trainee, Department of Otorhinolaryngology, Vivekananda Institute of Medical Sciences, Kolkata, West Bengal, India, e-mail: sachana18@gmail.com
Collaural fistula (Cervical-aural) is the least common first branchial cleft anomaly in which there is a communicating fistulous tract starting from a point at the angle of mandible and anterior border of sternocleidomastoid muscle. It sometimes traverses the parotid gland and ends as an opening in the floor of the external auditory canal at the level of the bony-cartilaginous junction or its cartilaginous portion. The relationship of the tract with the facial nerve is variable and it may lie medial, lateral or run through the branches of the facial nerve. Due to its varied clinical manifestations and rarity, misdiagnosis is common which further leads to inadequate and inappropriate management. Surgical excision is the treatment modality of choice. The aim of the treatment is complete removal of the lesion with preservation of the facial nerve if possible and reconstruction of the otological structures if necessary. Accurate diagnosis and management at primary presentation needs to be advocated to reduce complications and recurrence.

\section{CASE REPORT}

A 55-year-old female was referred to us for further management of a recurrent discharging right ear associated with a preauricular swelling extending to the upper neck since childhood (Fig. 1). She had undergone numerous incision and drainage procedures and also excision of cystic mass from the external auditory canal under local anesthesia. She was otherwise healthy.

On examination, there was a small cutaneous opening of size $2 \times 2 \mathrm{~mm}$ in the right submandibular region (Fig. 1). The surrounding skin was scarred and fibrosed due to previous infections. A small fistulous opening was also noted at the floor of the cartilaginous portion of the

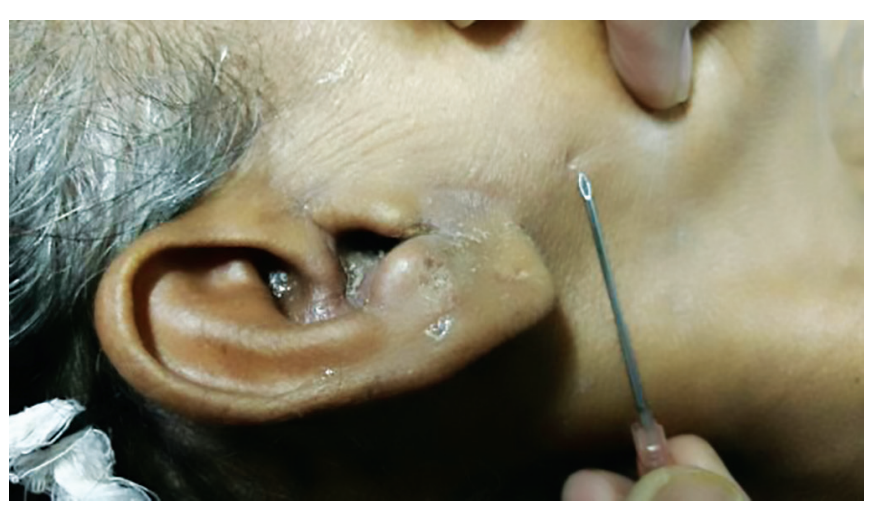

Fig. 1: The cervical opening of the collaural fistula 


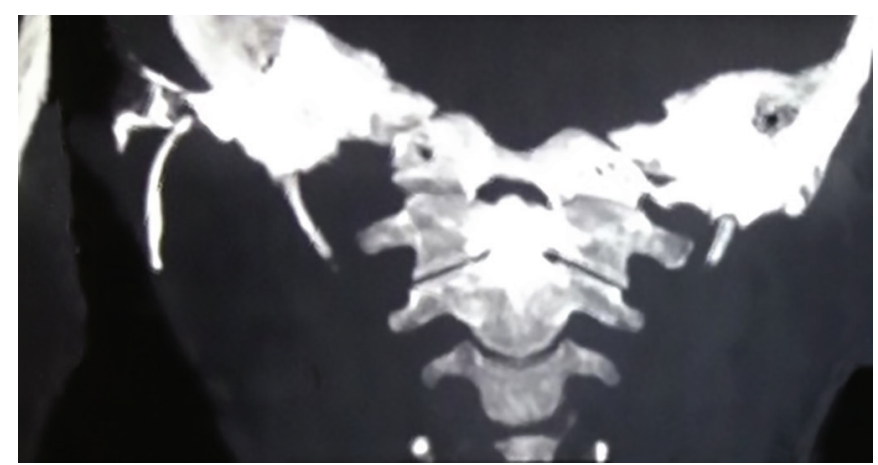

Fig. 2: CT fistulogram with coronal reconstruction showing the fistulous tract passing lateral to the parotid tissue

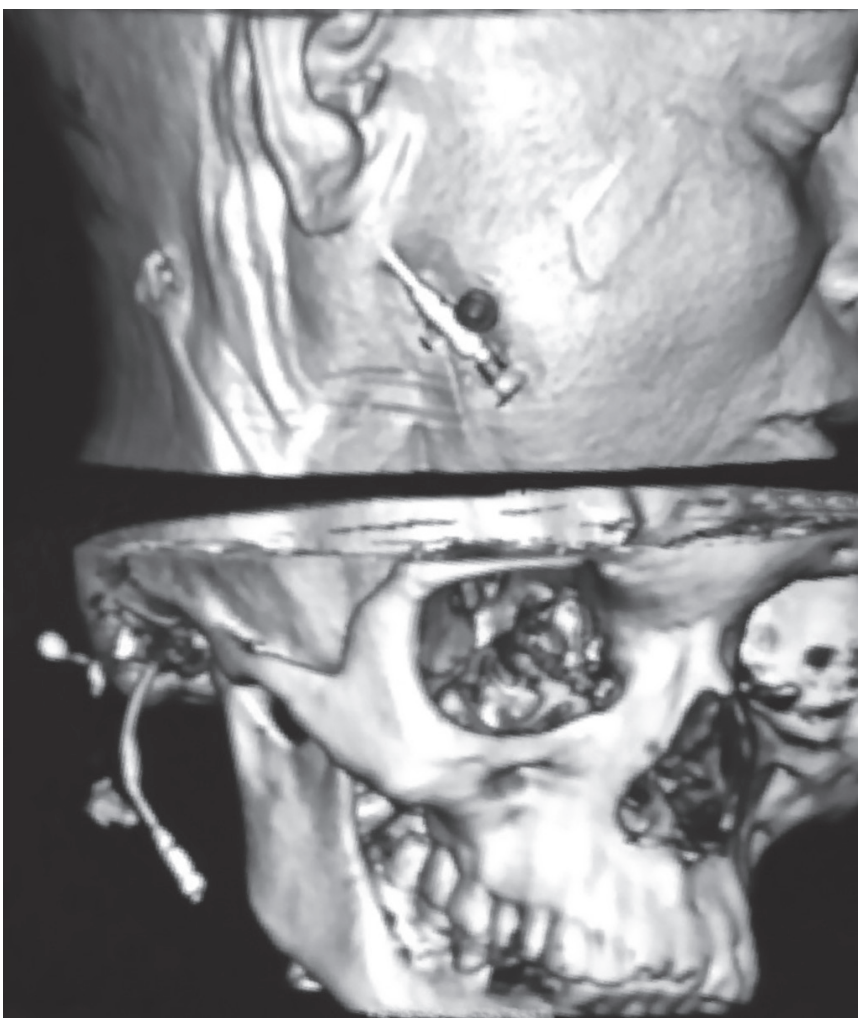

Fig. 3: CT fistulogram with 3D reconstruction showing the fistulous tract from the cervical region to the opening in the floor of the EAC

right external auditory canal covered with granulation tissue. The tympanic membrane appeared normal. A diagnosis of first branchial cleft fistula also called collaural fistula was made clinically.

CT fistulogram scans showed a wide fistulous tract connecting the cartilaginous portion of the right external ear canal and the upper lateral neck (Figs 2 and 3). It was situated lateral to the capsule of the parotid gland. During surgical exploration, methylene blue was injected through the lower neck skin opening to facilitate identification of the tract intraoperatively. A modified parotidectomy incision was given enclosing the neck opening with an elliptical incision (Fig. 4). The tract was traced and dissected from the surrounding tissue and followed from below upward till its opening in the external auditory canal (Figs 5A and B). The tragal pointer was identi-

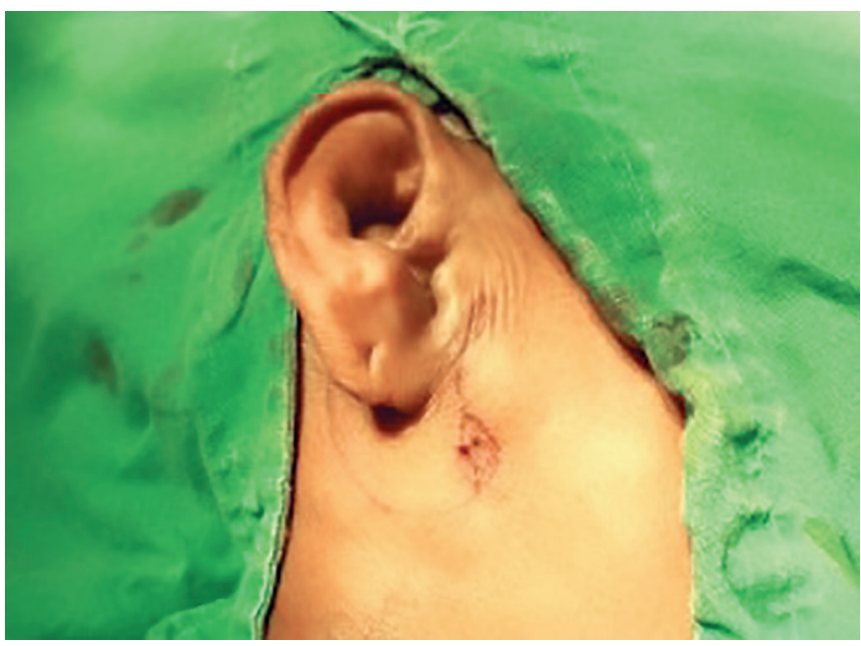

Fig. 4: Marking of the modified parotidectomy incision elliptically enclosing the cervical opening of the collaural fistula
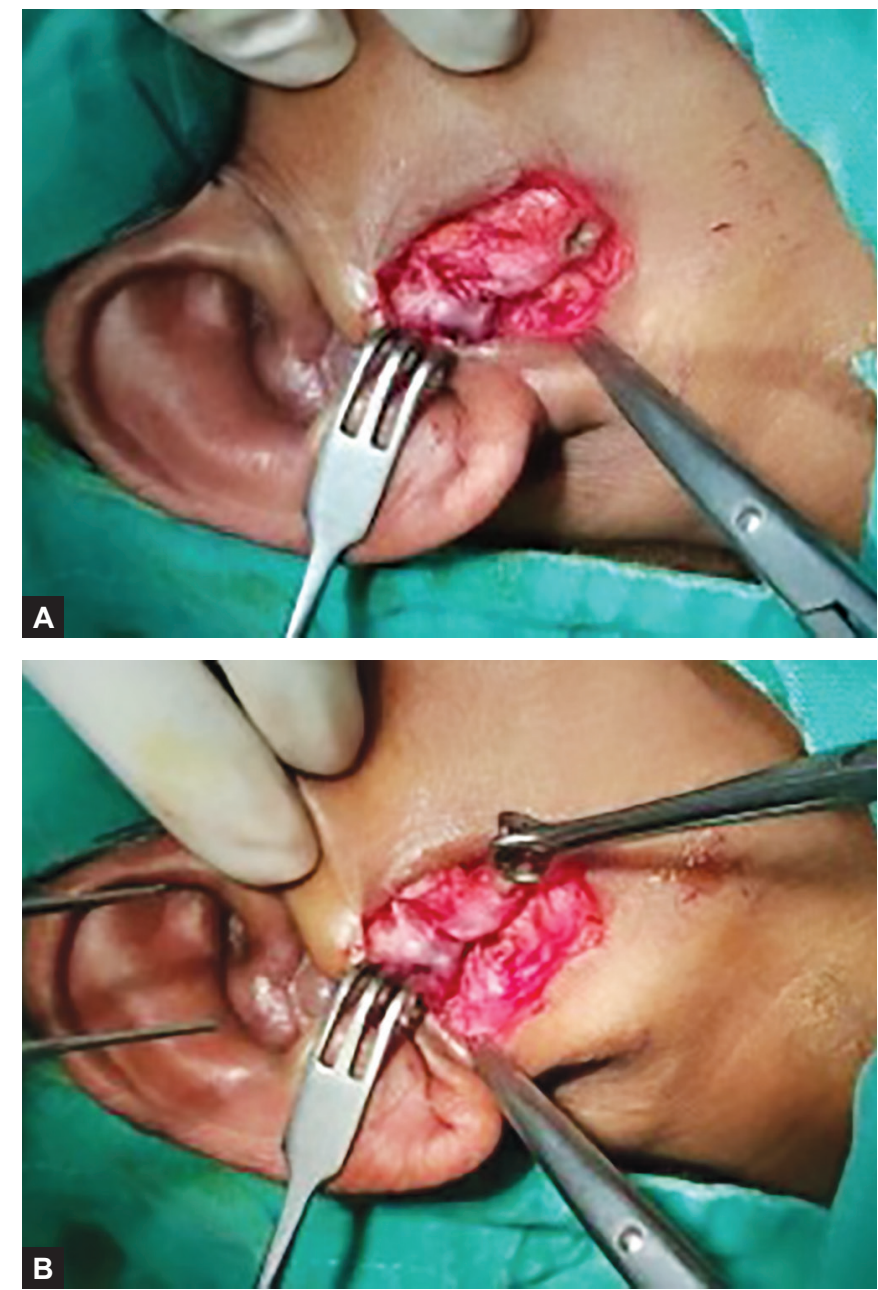

Figs 5A and B: Intraoperative picutures showing the excision of the fistulous tract

fied and the fistulous tract was noted to run lateral to it and thus superficial to the facial nerve. The tract was completely excised from lower skin opening upto the external auditory canal with a small cuff of external ear canal cartilage, granulation tissue and skin. The skin in the external auditory canal could be closed with primary 
suturing. Medicated aural packing was done. The postoperative recovery was uneventful. Regular dressing was done postoperatively.

Histological examination of excised specimen confirmed that it was lined by squamous epithelium with adnexal structures.

\section{DISCUSSION}

\section{Embryology}

The human embryo develops with the appearance of 6 pairs of branchial arches during the fourth week of intrauterine life. Branchial arches are derived from a Greek word "branchia" meaning gill owing to its resemblance to the gills of certain species of fish. These branchial arches are further separated by 5 clefts (ectoderm) externally and 5 pouches (endoderm) internally. By the seventh week of development, the arches fuse and clefts are obliterated to give rise to various structures. The structures derived from the first branchial cleft are cavum conchae, the EAC and the external layer of the tympanic membrane. First branchial cleft anomalies are a result of incomplete fusion of ventral portion of the first and second arches. During development, closure of the clefts is concurrent with the emergence of developing parotid gland and migration of the facial nerve, which originate from the second branchial arch; thus first branchial cleft anomalies are typically closely related to these structures. ${ }^{3}$ The chance of malformations occurring nearer to the ear and parotid is greater than that occurring at the hyoid region, as obliteration of the clefts proceeds from ventral to dorsal. The lesions normally have a close and variable relationship with parotid and facial nerve presumably because of temporal differences during development. ${ }^{4}$

\section{Classification}

In 1971, Arnot $^{5}$ proposed an anatomic classification in which he designated Type I anomalies as defects in the parotid region, appearing during early or middle adult life and Type II as those that appear in the anterior cervical triangle with a communicating tract to the external auditory canal usually developing during childhood.

In 1972, Work ${ }^{6}$ outlined a histological classification. Type 1 anomaly is a defect of ectodermal origin, arising from duplication of the membranous external auditory canal. Clinically, they appear as soft cysts lined by squamous epithelium. It can have a tract running medial and parallel to the external auditory canal, superior to the facial nerve and ending in a cul-de-sac on a bony plate at the level of the mesotympanum. Type 2 defects are ectodermal and mesodermal in origin, containing skin with adnexal structures as well as cartilage. They are present as cyst, sinus, fistula or combination. They are associated with a sinus/fistula opening in the region of the submandibular triangle, extending superiorly through the parotid gland toward the floor of the external auditory canal at the level of the bony-cartilaginous junction or the cartilaginous portion.

In 1980, Oslen et al. ${ }^{1}$ classified the defects as cysts, sinuses or fistulas.

\section{Clinical Presentation}

The mean age of presentation of first branchial cleft anomalies is 18.9 yrs, though it is congenital in origin. ${ }^{2}$

Due to its rarity and overlapping clinical symptoms, a patient with a branchial cleft anomaly may present to an otologist, head and neck surgeon, pediatric otolaryngologist and even to a general pediatrician or surgeon.

\section{To an Otologist}

Chronic otorrhrea with absence of otitis media is the most frequently encountered presentation. A sinus/ fistula opening in the external auditory canal is present in only $44 \%$ of patients, and even if such an opening exists, it may not necessarily appear obvious. ${ }^{7,8}$ It can also be associated with cholesteatoma and aural atresia as reported by Yalcin et al. ${ }^{9}$

In a study by Sichel et $\mathrm{al}^{8}$ on 3 cases, he reported that 2 of them had associated myringeal web, an epidermal structure that extends from the floor of the external auditory canal to the umbo of the tympanic membrane. Triglia et al, 7 from his study on larger series, concluded that it was found in only $10 \%$ of the patients.

\section{To the General Physician/Surgeon}

Patients most commonly present with a swelling in the cervical region (35\%), parotid (35\%), or preauricular region (24\%). ${ }^{9}$ It is often associated with infections and recurrent discharge. Pochet's triangle is an anatomical triangle where first branchial cleft cysts, sinuses and fistulas were typically located. ${ }^{8}$ The boundaries of the triangle comprises of the external auditory canal above, the mental region anteriorly and the hyoid bone inferiorly. Therefore, an inflammatory process in the region of the Pochet's triangle should immediately raise the index of suspicion.

Solares et al. ${ }^{4}$ reported a study on 10 patients with branchial cleft anomalies and stated that 7 out of 10 lesions ran medial to the facial nerve, 2 were lateral and 1 ran between the branches. Fistulas have a tendency to run deep into the nerve whereas sinus facts tend to run lateral to it. 


\section{To the Pediatrician}

As the branchial cleft anomalies are congenital in origin, Type II first branchial cleft cysts often present in young children. In a study conducted by Stokroos and Manni, ${ }^{11}$ 8 out of 18 patients presented at less than 10 years of age. Agenesis of the parotid can be associated and is suspected if a dimple anterior to the tragus is present.

Identification of facial nerve intraoperatively in younger children can be difficult due to its more superficial course and necessary modifications in the surgical technique should be undertaken.

\section{Management}

Rarity and diverse presentations of branchial cleft anomalies often lead to misdiagnosis and delay in accurate treatment. Triglia et al noted a delay of 3.5 years between the time of initial presentation and that when adequate treatment was received, with almost $50 \%$ of patients having a history of unsuccessful treatment. ${ }^{7}$ Accurate diagnosis is key to successful treatment, especially at primary presentation. The resultant recurrent infections and repeated surgery lead to increased scarring and higher risk of iatrogenic facial palsy during its subsequent surgical removal. ${ }^{7}$

Imaging studies have significant role in aiding diagnosis. CT scan can confirm the diagnosis by showing the tract near the external auditory canal to be wide and surrounded by cartilage. A CT fistulogram further helps to delineate the tract better and also to ascertain whether the lesion is medial, lateral or through the branches of the facial nerve.

A Type I cyst can be removed while still keeping the epidermal skin of the meatus intact via a retroauricular incision. ${ }^{10}$ Work recommended marsupialization of this cyst through the external ear canal. For a Type II lesion, early identification of the facial nerve at the stylomastoid foreman is recommended. ${ }^{10}$ If this part is affected by disease, identifying the facial nerve proximally in temporal bone and tracing it distally may be the safest option. Should the sinus/fistula opening involve the external auditory canal, it is removed with skin and cartilage. Primary closure is normally possible but if more than
$30 \%$ of the circumference of the external auditory canal is denuded, split-thickness skin grafting and stunting are recommended. If the tympanic membrane or middle ear structures are involved, reconstructive otological surgery may be necessary.

\section{CONCLUSION}

The rare and diverse clinical manifestations of branchial cleft anomalies make it prone to misdiagnosis and eventually inadequate treatment. The condition can be easily overlooked if the clinician adopts a compartmentalized speciality/subspecialist approach and fails to see the full clinical picture. Surgical dexterity should be available to excise the lesion completely, preserving all the branches of facial nerve if possible and reconstructive surgery if necessary.

\section{REFERENCES}

1. Olsen KD, Maragos NE, Weiland LH. First branchial cleft anomalies. Laryngoscope. 1980 Mar;90(3):423-436.

2. D'Souza AR, Uppal HS, De R, Zeitoun H. Updating concepts of first branchial cleft defects: a literature review. Int J Pediatr Otorhinolaryngol. 2002 Feb 1;62(2):103-109.

3. Benson MT, Dalen K, Mancuso A, Kerr HH, Cacciarelli A, Mafee MF. Congenital anomalies of the branchial apparatus: embryology and pathologic anatomy. Radiographics. 1992 Sep;12(5):943-960.

4. Solares C A, Chan J, Koltai P J. Anatomical variations of the facial nerve in rst branchial cleft anomalies. Arch Otolaryngol. 2003; 129(3):351-355

5. Arnot R S.Defects of the first branchial cleft. South Afr J Surg. 1971;9(2): 93-98.

6. Work WP. Newer concepts of first branchial cleft defects. Laryngoscope. 1972 Sep;82(9):1581-1593.

7. Triglia JM, Nicollas R, Ducroz V, Koltai PJ, Garabedian EN. First branchial cleft anomalies: a study of 39 cases and a review of the literature. Arch Otolaryngol-Head Neck Surg. 1998 Mar 1;124(3):291-295.

8. Sichel JY,Halperin D,Dano I,Dangoor E.Clinical update on type II first branchial cleft cysts. Laryngoscope 1998;108:1524-1527.

9. Yalcin S, Karlidag T, Kaygusuz I, Demirbag E. First branchial cleft sinus presenting with cholesteatoma and external auditory canal atresia. Int J Pediatr Otorhinolaryngol 2003;67:811-814.

10. Stokroos RJ, Manni JJ. The double auditory meatus - rare first branchial cleft anomaly: clinical presentation and treatment. Am J Otol 2000;21: 837-841. 